\title{
Landau quantization of two-dimensional heavy holes, energy spectrum of magnetoexcitons and Auger-recombination lines
}

\author{
I.V. Podlesny a,*, S.A. Moskalenko a, T. Hakioğlu ${ }^{\text {b,c }}$, A.A. Kiselyov ${ }^{\text {d, }}$ L. Gherciu ${ }^{\text {a }}$ \\ a Institute of Applied Physics, Academy of Sciences of Moldova, 5, Academiei str., MD-2028 Chisinau, Republic of Moldova \\ ${ }^{\mathrm{b}}$ Department of Physics, Bilkent University, 06800 Ankara, Turkey \\ c Institute of Theoretical and Applied Physics, 48740 Turunç, Marmaris, Muğla, Turkey \\ ${ }^{\mathrm{d}}$ State University of Civil Aviation, 38, Pilotov str., 196210 St. Petersburg, Russia
}

\section{H I G H L I G H T S}

- The Landau quantization of the two-dimensional heavy holes in electric field is studied.

- The Rashba spin-orbit coupling with third-order chirality term is taken into account.

- The shift of the Auger recombination line under the influence of the magnetic field is explained.

\section{A R T I C L E I N F O}

\section{Article history:}

Received 3 August 2012

Received in revised form

2 January 2013

Accepted 18 January 2013

Available online 29 January 2013

\begin{abstract}
A B S T R A C T
The Landau quantization of the two-dimensional (2D) heavy holes, its influence on the energy spectrum of 2D magnetoexcitons, as well as their optical orientation are studied. The Hamiltonian of the heavy holes is written in two-band model taking into account the Rashba spin-orbit coupling (RSOC) with two spin projections, but with nonparabolic dispersion law and third-order chirality terms. The most Landau levels, except three with $m=0,1,2$, are characterized by two quantum numbers $m-3$ and $m$ for $m \geq 3$ for two spin projections correspondingly. The difference between them is determined by the third-order chirality. Four lowest Landau levels (LLLS) for heavy holes were combined with two LLLs for conduction electron, which were taken the same as they were deduced by Rashba in his theory of spinorbit coupling (SOC) based on the initial parabolic dispersion law and first-order chirality terms. As a result of these combinations eight $2 \mathrm{D}$ magnetoexciton states were formed. Their energy spectrum and the selection rules for the quantum transitions from the ground state of the crystal to exciton states were determined. On this base such optical orientation effects as spin polarization and magnetoexciton alignment are discussed. The continuous transformation of the shake-up (SU) into the shake-down (SD) recombination lines is explained on the base of nonmonotonous dependence of the heavy hole Landau quantization levels as a function of applied magnetic field.
\end{abstract}

(c) 2013 Elsevier B.V. All rights reserved.

\section{Introduction}

The quantum states of a free spinless electron with parabolic dispersion law under the influence of a magnetic field were investigated by Landau [1]. This procedure is known as Landau quantization.

The Landau quantization of an electron with the spin taking into account the spin-orbit coupling (SOC) was firstly studied by Rashba [2] in the frame of two-band model. Its Hamiltonian has diagonal elements expressed through the initial parabolic dispersion law $\hbar^{2} k^{2} / 2 m$ and the nondiagonal elements containing the

\footnotetext{
* Corresponding author. Tel.: +373 22 738084; fax: +37322 738149 .

E-mail addresses: exciton@phys.asm.md, podlesniy@rambler.ru (I.V. Podlesny), hakioglu@bilkent.edu.tr (T. Hakioğlu).
}

first-order chirality terms $k_{ \pm}=k_{x} \pm i k_{y}$, where $\vec{k}$ is the wave vector of the conduction electron in a bulk crystal. The SOC and chirality terms in Ref. [2] are induced by the external electric field $E_{z}$ applied parallel to the magnetic field. The electron wave functions were written in a spinor form with two components corresponding to spin orientation along $z$ axis. The energy levels except one with $n=0$ are characterized by two quantum numbers $n$ and $n^{\prime}$ different for two spin projections. They differ by 1 in the case of first-order chirality terms. The method proposed by Rashba [2] was applied in Refs. [3,4] to describe the Landau quantization of two-dimensional (2D) heavy holes with nonparabolic initial dispersion law, two spin projections and third-order chirality terms proportional to $\left(k_{+}\right)^{3}$, as well as to the electron in the biased bilayer graphene with nonparabolic initial dispersion law, two pseudospin components and second-order chirality 
terms proportional to $\left(k_{ \pm}\right)^{2}$. In both cases the two-band models were applied and the differences between the numbers $m$ and $m^{\prime}$ were equal to 3 and 2 correspondingly.

The aim of our paper is to obtain new information in comparison with Ref. [3] concerning the 2D heavy holes and their Landau quantization levels in dependence on the magnetic field strength at different parameters of the initial nonparabolic dispersion law. These details influence on the electron structure of the 2D magnetoexcitons and determine the selection rules of the quantum transitions from the ground state of the crystal to exciton states.

The shake-up and shake-down recombination lines with the participation of the acceptor-bound trions $\mathrm{AX}^{+}$were studied.

\section{Landau quantization energy levels of $2 \mathrm{D}$ heavy holes}

The full Landau-Rashba Hamiltonian for 2D heavy holes was discussed in Ref. [3] following the formulas (13)-(20). It can be expressed through the Bose-type creation and annihilation operators $a^{\dagger}, a$ acting on the Fock quantum states $|n\rangle=\left(\left(a^{\dagger}\right)^{n} / \sqrt{n !}\right)|0\rangle$, where $|0\rangle$ is the vacuum state of harmonic oscillator. The Hamiltonian has the form

$\hat{H}_{h}=\hbar \omega_{c h}\left\{\left[\left(a^{\dagger} a+\frac{1}{2}\right)+\delta\left(a^{\dagger} a+\frac{1}{2}\right)^{2}\right] \hat{I}+i \beta 2 \sqrt{2}\left|\begin{array}{cc}0 & \left(a^{\dagger}\right)^{3} \\ -a^{3} & 0\end{array}\right|\right\}, \quad \hat{I}=\left|\begin{array}{ll}1 & 0 \\ 0 & 1\end{array}\right|$,

with the denotations

$\omega_{c h}=\frac{|e| H}{m_{h} c}, \quad \delta=\frac{\left|\delta_{h} E_{z}\right| \hbar^{4}}{l^{4} \hbar \omega_{c h}}, \quad \beta=\frac{\beta_{h} E_{z}}{l^{3} \hbar \omega_{c h}}, \quad l=\sqrt{\frac{\hbar c}{|e| H}}$.

The parameter $\delta_{h}$ is not well known, what permits to consider different variants mentioned below.

The exact solution of the Pauli-type Hamiltonian is described by the formulas (21)-(31) of Ref. [3] and has the spinor form

$\hat{H}_{h}\left|\begin{array}{l}f_{1} \\ f_{2}\end{array}\right|=E_{h}\left|\begin{array}{l}f_{1} \\ f_{2}\end{array}\right|, \quad f_{1}=\sum_{n=0}^{\infty} c_{n}|n\rangle, \quad f_{2}=\sum_{n=0}^{\infty} d_{n}|n\rangle$,

$\sum_{n=0}^{\infty}\left|c_{n}\right|^{2}+\sum_{n=0}^{\infty}\left|d_{n}\right|^{2}=1$.

First three solutions depend only on one quantum number $m$ with the values $0,1,2$ as follows:

$E_{h}(m=0)=\hbar \omega_{c h}\left(\frac{1}{2}+\delta\right), \quad \Psi(m=0)=\left|\begin{array}{c}|0\rangle \\ 0\end{array}\right|$,
$E_{h}(m=1)=\hbar \omega_{c h}\left(\frac{3}{2}+9 \delta\right), \quad \Psi(m=1)=\left|\begin{array}{c}|1\rangle \\ 0\end{array}\right|$,
$E_{h}(m=2)=\hbar \omega_{c h}\left(\frac{5}{2}+25 \delta\right), \quad \Psi(m=2)=\left|\begin{array}{c}|2\rangle \\ 0\end{array}\right|$.

All another solutions with $m \geq 3$ depend on two quantum numbers $(m-5 / 2)$ and $(m+1 / 2)$ and have the general expression

$$
\begin{aligned}
\varepsilon_{h}^{ \pm}\left(m-\frac{5}{2}, m+\frac{1}{2}\right)= & \frac{E_{h}^{ \pm}(m-5 / 2 ; m+1 / 2)}{\hbar \omega_{c h}} \\
= & (m-1)+\frac{\delta}{2}\left[(2 m+1)^{2}+(2 m-5)^{2}\right] \\
& \pm\left(\left(\frac{3}{2}+\frac{\delta}{2}\left[(2 m+1)^{2}-(2 m-5)^{2}\right]\right)^{2}\right. \\
& \left.+\beta^{2} m(m-1)(m-2)\right)^{1 / 2}, m \geq 3 .
\end{aligned}
$$

The corresponding wave functions for $m=3$ and $m=4$ are

$\Psi_{h}^{ \pm}(m=3)=\left|\begin{array}{c}c_{3}|3\rangle \\ d_{0}|0\rangle\end{array}\right|$ and $\Psi_{h}^{ \pm}(m=4)=\left|\begin{array}{c}c_{4}|4\rangle \\ d_{1}|1\rangle\end{array}\right|$.
They depend on the coefficients $c_{m}$ and $d_{m-3}$, which obey to the equations

$c_{m}\left(m+\frac{1}{2}+\delta(2 m+1)^{2}-\varepsilon_{h}\right)=-i \beta 2 \sqrt{2} \sqrt{m(m-1)(m-2)} d_{m-3}$,

$d_{m-3}\left(m-\frac{5}{2}+\delta(2 m-5)^{2}-\varepsilon_{h}\right)=i \beta 2 \sqrt{2} \sqrt{m(m-1)(m-2)} c_{m}$,

$\left|c_{m}\right|^{2}+\left|d_{m-3}\right|^{2}=1$.

There are two different solutions $\varepsilon_{h}^{ \pm}(m)$ at a given value of $m \geq 3$ and two different pairs of the coefficients $\left(c_{m}^{ \pm}, d_{m-3}^{ \pm}\right)$.

The dependences of the parameters $\omega_{c h}, \beta$ and $\delta$ on the electric and magnetic fields strengths may be represented for the GaAs-type quantum wells as follows $H=y \mathrm{~T} ; E_{z}=x \mathrm{kV} / \mathrm{cm} ; m_{h}=0.25 m_{0}$; $\hbar \omega_{c h}=0.4 y \mathrm{meV} ; \quad \beta=1.062 \times 10^{-2} x \sqrt{y} ; \quad \delta=10^{-4} \mathrm{Cxy} \quad$ with unknown parameter $C$, which will be varied in more large interval of values. We cannot neglect the parameter $C$ putting it equal to zero, because in this case, as was argued in Ref. [3] formula (10), the lower spinor branch of the heavy hole dispersion law

$E_{h}^{-}\left(k_{\|}\right)=\frac{\hbar^{2} \vec{k}_{\|}^{2}}{2 m_{h}}-\left|\frac{\beta_{h} E_{z}}{2}\right|\left|\vec{k}_{\|}\right|^{3}$

has an unlimited decreasing, deeply penetrating inside the semiconductor energy gap at great values of $\left|\vec{k}_{\|}\right|$. To avoid this unphysical situation the positive quartic term $\left|\delta_{h} E_{z}\right| \vec{k}_{\|}^{4}$ was added in the starting Hamiltonian. The new dependences will be compared with the drawings calculated in Fig. 2 of Ref. [3] in the case $E_{z}=10 \mathrm{kV} / \mathrm{cm}$ and $C=10$. Four lowest Landau levels (LLLs) for heavy holes are selected as in Ref. [3]. In addition to them we will study else three levels as follows:

$E_{h}\left(R_{1}\right)=E_{h}^{-}\left(\frac{1}{2}, \frac{7}{2}\right), \quad E_{h}\left(R_{2}\right)=E_{h}(m=0)$,

$E_{h}\left(R_{3}\right)=E_{h}^{-}\left(\frac{3}{2}, \frac{9}{2}\right), \quad E_{h}\left(R_{4}\right)=E_{h}(m=1)$,

$E_{h}\left(R_{5}\right)=E_{h}^{-}\left(\frac{5}{2}, \frac{11}{2}\right), \quad E_{h}\left(R_{6}\right)=E_{h}(m=2)$,

$E_{h}\left(R_{7}\right)=E_{h}^{-}\left(\frac{7}{2}, \frac{13}{2}\right)$.

Their dependences on the magnetic field strength are represented in Figs. 1 and 2 at different parameters $x$ and $C$.

The general view of the lower branches $E_{h}^{-}\left(m-\frac{5}{2}, m+\frac{1}{2}\right)$ of the heavy hole Landau quantization levels with $m \geq 3$ as a function of the magnetic field strength are represented in Fig. 1a following the formula (5). The upper branches have more simple monotonous behavior and are drawn in Fig. 1b together with some curves of the lower branches. All the lower branches in their initial parts have a linear increasing behavior up till they achieve the maximal values succeeded by the minimal values in the middle parts of their evolutions being transformed in the final quadratic increasing dependences. The values of the magnetic field strength corresponding to the minima and to the maxima decrease with the increasing of the number $m$. These peculiarities can be compared with the case of Landau quantization of the 2D electron in the biased bilayer graphene described in Ref. [4]. The last case is characterized by the initial dispersion law without parabolic part and by second-order chirality terms. They both lead to dependences on the magnetic field strength for the lower dispersion branches with sharp initial decreasing parts and minimal values succeeded by the quadratic increasing behavior. The differences between the initial dispersion laws and chirality terms in two cases of bilayer graphene and heavy holes lead to different intersections and degeneracies of the Landau levels. Fig. 2 shows that the change of the parameter $C$ at a given parameter $E_{z}$ (or vice versa) shifts significantly on the energy scale the lower branches of the heavy hole Landau levels. It can be observed in all four sections of Fig. 2. But there is a special case in Section $2 \mathrm{~b}$, where the degeneracy of the levels $R_{1}, R_{3}, R_{5}$ and $R_{7}$ take place in the range of magnetic field strength 5-10 T. More so, 

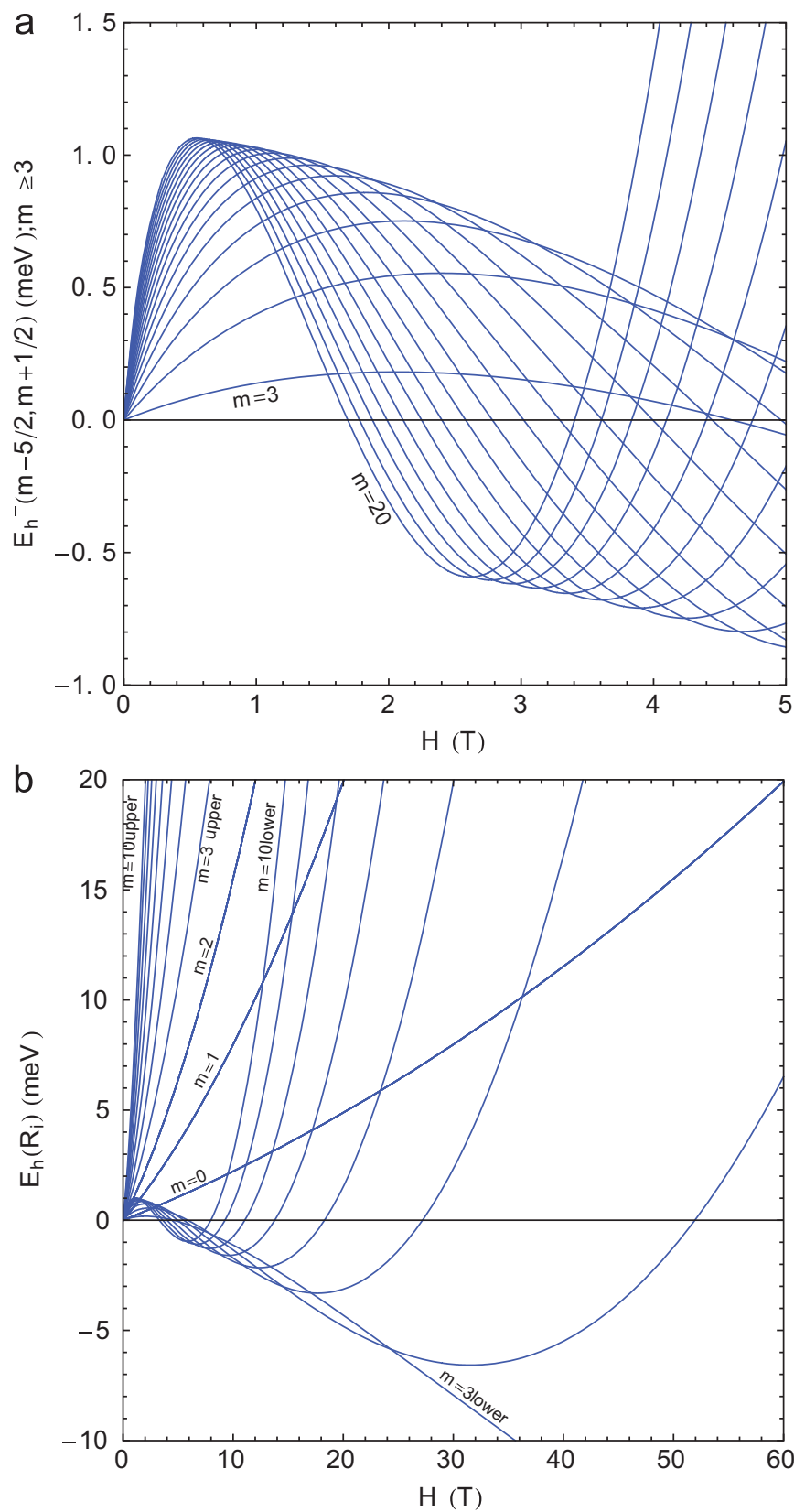

Fig. 1. (a) The lower branches of the heavy hole Landau quantization levels $E_{h}^{-}(m-5 / 2 ; m+1 / 2)$ for $m \geq 3$ at the parameters $E_{z}=10 \mathrm{kV} / \mathrm{cm}$ and $C=5.5$; (b) the general view of the all heavy hole Landau quantization levels with $m=0,1, \ldots, 10$ at the same parameters $E_{z}$ and $C$.

the degeneracy of the levels $R_{1}$ and $R_{3}$ persists to exist even in a more large interval as 5-20 T.

The degeneracy of two lowest Landau levels (LLLs) in biased bilayer graphene was suggested to explain the experimental results related with the fractional quantum Hall effects $[5,6]$. Meanwhile the degeneracy of two LLLs in biased bilayer graphene in Ref. [4] was revealed only near the intersection point at a given value of the magnetic field strength. To obtain a more complete and wide degeneracy in the calculations concerning the biased bilayer graphene one could employ a mixed model using the results reflected in Fig. 2.

The Landau quantization of the electrons and holes determine the energies of the optical band-to-band quantum transitions as well as of the magnetoexcitons creation. The band-to-band quantum transitions can be discussed in a more large range of parameters because they do not need the knowledge about the ionization potentials of the magnetoexcitons. By this reason the quantum transitions from the ground state of the crystal to the exciton states will be confined by the quantum numbers $m \leq 4$. The heavy hole LLLs denoted as $\left(h R_{j}\right)$ with $j=1,2,3,4$ were combined with two LLLs of the conduction electron $\left(e R_{i}\right)$ with $i=1,2$. These combinations have the energies of the band-to-band transitions $E_{c v}\left(F_{n}\right)$ accounted from the semiconductor energy gap $E_{g}$ equal to

$E_{c v}\left(F_{n}\right)-E_{g}=E_{e}\left(R_{i}\right)+E_{h}\left(R_{j}\right)$.

They are represented in Fig. 3 in dependence on the magnetic field strength for the parameter $E_{z}=10 \mathrm{kV} / \mathrm{cm}$ and two values of the coefficient $C: 2.6$ (a) and 5.65 (b). The lines on the drawings are doubled because the differences between the electron LLs $\left(e R_{1}\right)$ and $\left(e R_{2}\right)$ are too small and the positions of the curves are mainly determined by the structure of the heavy hole LLs.

\section{Energy spectrum and selection rules for 2D magnetoexcitons}

The four LLLs for 2D heavy holes were combined with two LLLs for 2D conduction electrons giving rise to eight 2D magnetoexciton states $F_{n}$ with $n=1,2, \ldots, 8$. They were determined by the formulas (37)-(42) of Ref. [3]. The creation energies of the eight magnetoexciton states are

$E_{e x}\left(F_{n}, k\right)=E_{c v}\left(F_{n}\right)-I_{e x}\left(F_{n}, k\right)$,

where

$$
\begin{aligned}
& E_{c v}\left(F_{1}\right)-E_{g}=E_{e}\left(R_{1}\right)+E_{h}\left(R_{1}\right), \\
& \begin{aligned}
I_{e x}\left(F_{1}, \vec{k}\right)= & I_{e x}\left(e, R_{1} ; h, R_{1} ; \vec{k}\right) \\
= & \left|a_{0}\right|^{2}\left|d_{0}\right|^{2} I_{e x}^{(0,0)}(\vec{k})+\left|a_{0}\right|^{2}\left|c_{3}\right|^{2} I_{e x}^{(0,3)}(\vec{k}) \\
& +\left|d_{0}\right|^{2}\left|b_{1}\right|^{2} I_{e x}^{(0,1)}(\vec{k})+\left|b_{1}\right|^{2}\left|c_{3}\right|^{2} I_{e x}^{(1,3)}(\vec{k}),
\end{aligned}
\end{aligned}
$$

$E_{c v}\left(F_{2}\right)-E_{g}=E_{e}\left(R_{2}\right)+E_{h}\left(R_{1}\right)$,

$I_{e x}\left(F_{2}, \vec{k}\right)=I_{e x}\left(e, R_{2} ; h, R_{1} ; \vec{k}\right)=\left|d_{0}\right|^{2} I_{e x}^{(0,0)}(\vec{k})+\left|c_{3}\right|^{2} I_{e x}^{(0,3)}(\vec{k})$,

$E_{c v}\left(F_{3}\right)-E_{g}=E_{e}\left(R_{1}\right)+E_{h}\left(R_{2}\right)$,

$$
\begin{aligned}
I_{e x}\left(F_{3}, \vec{k}\right) & =I_{e x}\left(e, R_{1} ; h, R_{2} ; \vec{k}\right) \\
& =\left|a_{0}\right|^{2} I_{e x}^{(0,0)}(\vec{k})+\left|b_{1}\right|^{2} I_{e x}^{(0,1)}(\vec{k}),
\end{aligned}
$$

$E_{c v}\left(F_{4}\right)-E_{g}=E_{e}\left(R_{2}\right)+E_{h}\left(R_{2}\right)$,

$I_{e x}\left(F_{4}, \vec{k}\right)=I_{e x}\left(e, R_{2} ; h, R_{2} ; \vec{k}\right)=I_{e x}^{(0,0)}(\vec{k})$,

$E_{c v}\left(F_{5}\right)-E_{g}=E_{e}\left(R_{1}\right)+E_{h}\left(R_{3}\right)$

$$
\begin{aligned}
I_{e x}\left(F_{5}, \vec{k}\right)= & I_{e x}\left(e, R_{1} ; h, R_{3} ; \vec{k}\right) \\
= & \left|a_{0}\right|^{2}\left|c_{4}\right|^{2} I_{e x}^{(0,4)}(\vec{k})+\left|a_{0}\right|^{2}\left|d_{1}\right|^{2} I_{e x}^{(0,1)}(\vec{k}) \\
& +\left|b_{1}\right|^{2}\left|c_{4}\right|^{2} I_{e x}^{(1,4)}(\vec{k})+\left|b_{1}\right|^{2}\left|d_{1}\right|^{2} I_{e x}^{(1,1)}(\vec{k}),
\end{aligned}
$$

$E_{c v}\left(F_{6}\right)-E_{g}=E_{e}\left(R_{2}\right)+E_{h}\left(R_{3}\right)$,

$I_{e x}\left(F_{6}, \vec{k}\right)=I_{e x}\left(e, R_{2} ; h, R_{3} ; \vec{k}\right),=\left|c_{4}\right|^{2} I_{e x}^{(0,4)}(\vec{k})+\left|d_{1}\right|^{2} I_{e x}^{(0,1)}(\vec{k})$,

$E_{c v}\left(F_{7}\right)-E_{g}=E_{e}\left(R_{1}\right)+E_{h}\left(R_{4}\right)$,

$I_{e x}\left(F_{7}, \vec{k}\right)=I_{e x}\left(e, R_{1} ; h, R_{4} ; \vec{k}\right)=\left|a_{0}\right|^{2} I_{e x}^{(0,1)}(\vec{k})+\left|b_{1}\right|^{2} I_{e x}^{(1,1)}(\vec{k})$,

$E_{c v}\left(F_{8}\right)-E_{g}=E_{e}\left(R_{2}\right)+E_{h}\left(R_{4}\right)$, 

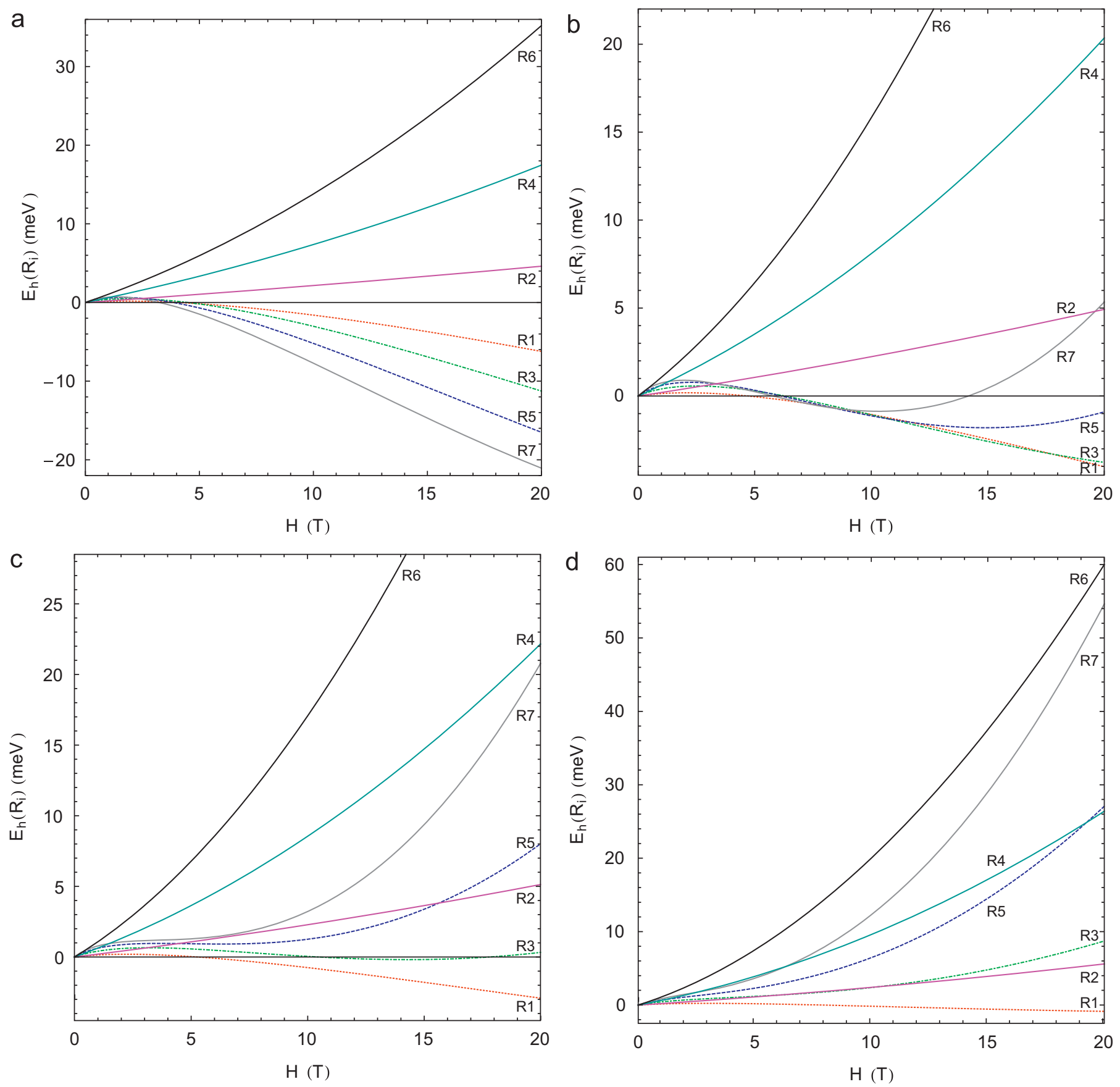

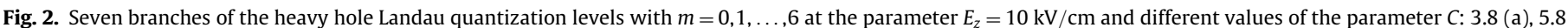
(b), 7.05 (c) and 10 (d).

$I_{e x}\left(F_{8}, \vec{k}\right)=I_{e x}\left(e, R_{2} ; h, R_{4} ; \vec{k}\right)=I_{e x}^{(0,1)}(\vec{k})$.

The ionization potentials $I_{e x}\left(e, R_{i} ; h, R_{j} ; \vec{k}\right)$ are determined by the Coulomb electron-hole interaction integrals

$I_{e x}\left(e, R_{i} ; h, R_{j} ; \vec{k}\right)=\frac{1}{N} \sum_{p, s} e^{i k_{y} s l^{2}} F_{e-h}\left(e, R_{i}, p ; h, R_{j}, k_{x}\right.$

$$
\left.-p ; e, R_{i}, p-s ; h, R_{j}, k_{x}+s-p\right) \text {. }
$$

The most of them were calculated in Refs. [3,7], whereas the values $I_{e x}^{(0,4)}(\vec{k})$ and $I_{e x}^{(1,4)}(\vec{k})$ are determined below. Their dependences on the magnetic field strength $H$ can be demonstrated only in the range $H \geq 7 \mathrm{~T}$, because the magnetoexcitons do exist only when the cyclotron energy is greater than the Coulomb interaction between electrons and holes. The criterion $I_{l}<\hbar \omega_{c}$ is used.
The most interest represents eight lowest exciton energy levels in the point $k=0$, where the optical quantum transitions take place. Four of them, namely $E_{e x}\left(F_{1}, 0\right), E_{e x}\left(F_{2}, 0\right), E_{e x}\left(F_{3}, 0\right)$, $E_{e x}\left(F_{4}, 0\right)$, were discussed in Ref. [3]. Another four exciton levels such as $F_{5}, F_{6}, F_{7}$ and $F_{8}$ we calculated below and are represented in Figs. 4 and 5 .

In Fig. 4a and b the exciton energy levels are drawn in dependence on the magnetic field strength $H$ at the same parameter $E_{z}=10 \mathrm{kV} / \mathrm{cm}$ but at different values of the coefficient $C$ equal to 3.35 and 5.65 correspondingly. The arrangement of the exciton energy levels on the energy scale in the order from the lower to upper values depends on the range of magnetic field strength. In Fig. $4 \mathrm{a}$ in the range $7-13 \mathrm{~T}$ their disposition is as follows: the dipole active state $F_{1}\left(\right.$ d.a.), the forbidden state $F_{2}(f$.), 

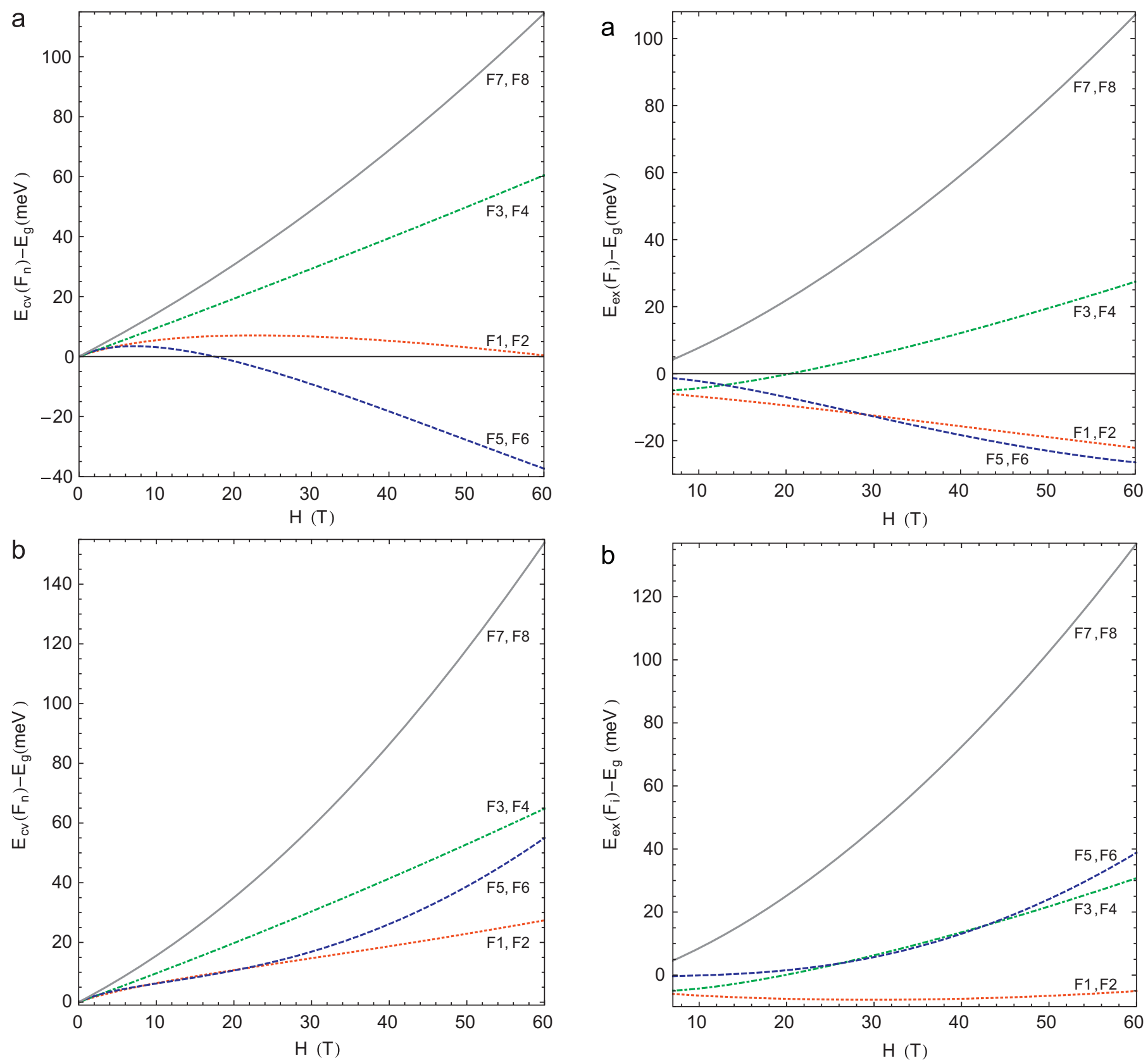

Fig. 3. The energies $E_{c v}\left(F_{n}\right)$ of the band-to-band quantum transitions starting from the LLLs of the heavy holes with the creation of the conduction electrons on the nearly degenerate two LLLs at the parameter $E_{z}=10 \mathrm{kV} / \mathrm{cm}$ and two values of the constant $C=2.6$ (a) and 5.65 (b).

the quadrupole-active state $F_{3}(q . a$.$) , the dipole active state$ $F_{4}$ (d.a.), the quadrupole-active state $F_{5}(q . a$.), the forbidden state $F_{6}\left(f\right.$.), the dipole active state $F_{7}($ d.a. $)$ and the quadrupole-active state $F_{8}$ (q.a.). There are three dipole-active states $\left(F_{1}, F_{4}, F_{7}\right)$, three quadrupole-active states $\left(F_{3}, F_{5}, F_{8}\right)$ and two forbidden states $\left(F_{2}, F_{6}\right)$. In the middle range of $H=13-27 \mathrm{~T}$ their distribution is another as follows: $F_{1}$ (d.a.), $F_{2}(f),. F_{5}$ (q.a.), $F_{6}(f),. F_{3}$ (q.a.), $F_{4}($ d.a.), $F_{7}$ (d.a.), $F_{8}$ (q.a.). In the last range of $H=(27-60) \mathrm{T}$ the third ordering of the exciton levels does exist: $F_{5}(q . a),. F_{6}(f),. F_{1}(d . a$.$) ,$ $F_{2}(f),. F_{3}$ (q.a.), $F_{4}\left(\right.$ d.a.), $F_{7}$ (d.a.), $F_{8}$ (q.a.). The scheme of exciton energy levels at a greater values $C=5.65$ represented in Fig. $4 \mathrm{~b}$ in the range 7-24 $\mathrm{T}$ is the same as in Fig. $4 \mathrm{a}$ in the range 7-13 $\mathrm{T}$. In the range 24-46 T the two lowest exciton levels remain the same $F_{1}\left(d . a\right.$.) and $F_{2}(f$.) being succeeded by the four nearly degenerate levels $F_{3}$ (q.a.), $F_{4}\left(\right.$ d.a.), $F_{5}\left(q . a\right.$.) and $F_{6}(f$.). The remained exciton

Fig. 4. The exciton energy levels in dependence on the magnetic field strength $H$ at the parameter $E_{z}=10 \mathrm{kV} / \mathrm{cm}$ and two values of the coefficient $C: 3.35$ (a) and 5.65 (b).

levels $F_{7}$ (d.a.) and $F_{8}$ (q.a.) are well separated from the previous ones.

Figs. 4 and 5 show that the changes of the parameters $E_{z}$ and $C$ as well as of the magnetic field strength $H$ may essentially change the arrangements of the exciton energy levels on the energy scale as well as the intensities of the optical quantum transitions from the ground state of the crystal to the magnetoexciton states.

\section{Auger-recombination emission line of acceptor-bound trions}

The magneto-photoluminescence spectra of the two-dimensional hole gas (2DHG) in the presence of the photo-generated electrons in GaAs quantum wells (QWs) revealed many emission 

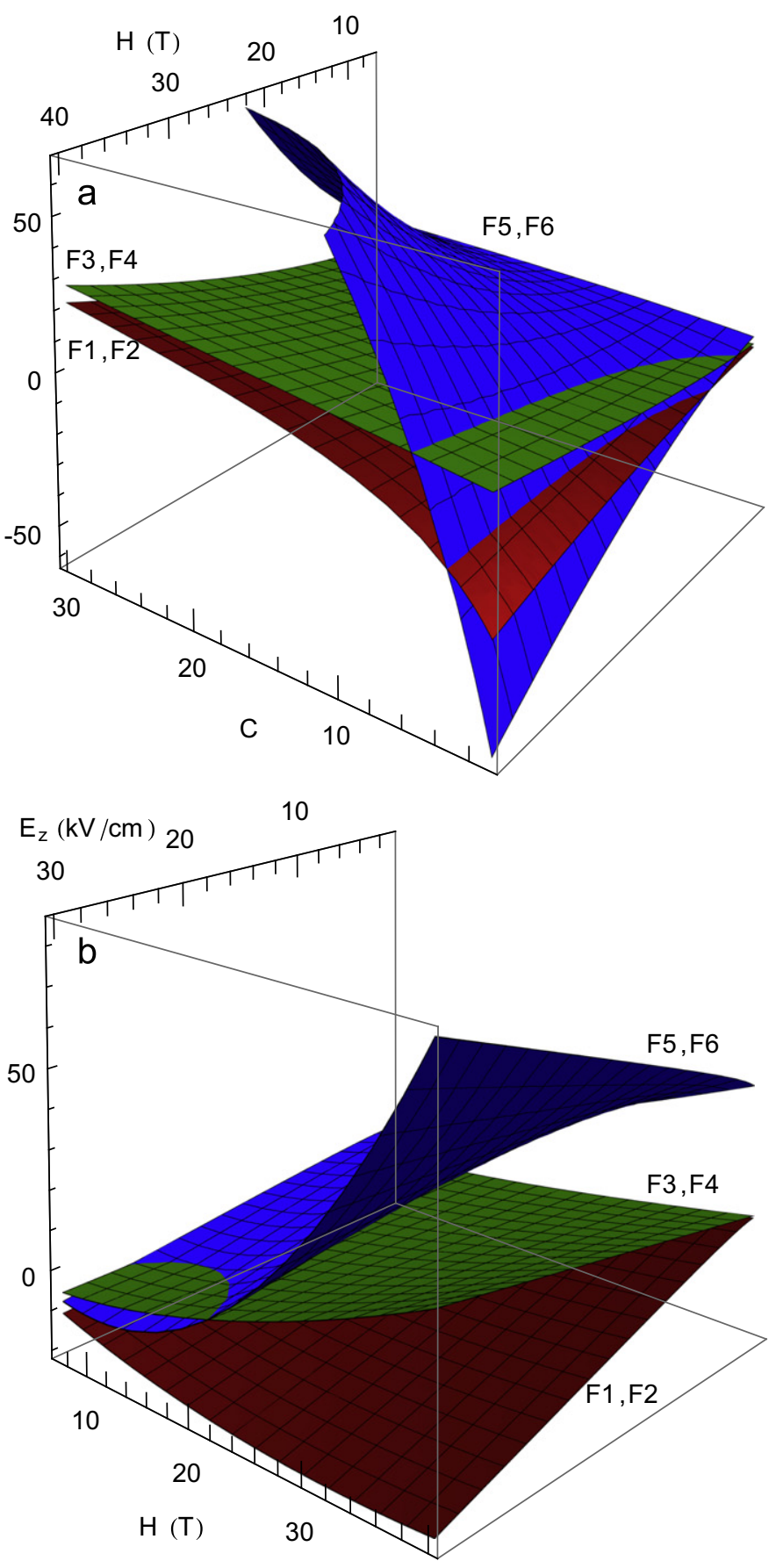

40

Fig. 5. Energy spectrum of the lower exciton energy levels with wave vector $k=0$ at a given parameter $E_{z}=10 \mathrm{kV} / \mathrm{cm}$ and different values of the coefficient $C$ and magnetic field strength $H(a)$, as well as at a given coefficient $C=10$ and different values of parameters $E_{z}$ and $H(b)$.

lines [8-10]. They correspond to the radiative recombinations of the electron-hole $(\mathrm{e}-\mathrm{h})$ pairs in different $\mathrm{e}-\mathrm{h}$ complexes such as free excitons $(\mathrm{X})$, positive trions $\left(\mathrm{X}^{+}\right)$and acceptor-bound trions $\left(\mathrm{AX}^{+}\right)$. The impurity complexes $\mathrm{AX}^{+}$revealed also the Augerrecombination lines. They appear when the $\mathrm{e}-\mathrm{h}$ annihilation is accompanied by the excitation or by de-excitation of a leftover acceptor-bound hole to higher or to lower Landau levels correspondingly. In the first case the Auger-recombination is known as shake-up (SU) process [11-15], whereas in the second variant it is named as shake-down (SD) process. The SU processes were detected experimentally and described in Refs. [11-15]. The evolution of the SU process into the SD process was described in Ref. [16]. The selection rules associated with translational symmetry of the QWs, as well as with the angular momentum conservation law in the photon emission preclude the Auger recombination of the free trions $[14,15]$. But they do not forbid the similar processes with participation of $\mathrm{AX}^{+}$complexes. In the case of spinless holes with parabolic dispersion law the distances between the adjacent levels equals to cyclotron energy $\hbar \omega_{c h}$. But the holes with nonparabolic dispersion law, with pseudo-spin components and with chirality terms in the presence of the strong external perpendicular electric field have completely another Landau-Rashba-type energy spectrum [3,4]. The conditions of the first type were encountered in the symmetric GaAs QWs investigated in Ref. [8,9]. Here the Auger-recombination line corresponding to SU process was detected. It was denoted as $\mathrm{AX}^{+} \mathrm{SU}$ and is represented in Fig. 6 reproduced from Ref. [8]. The experimental data [8] were obtained in the symmetric conditions in the absence of the one-sized doping and in the absence of the applied perpendicular electric field when the Rashba effect is not present.

The situation of the second type was met [10] in the asymmetric GaAs QWs subjected to the action of a strong perpendicular electric field caused by the one-sided doping. In these conditions Rashba spin-orbit coupling plays an important role $[3,4]$. In Ref. [10] an unusual Auger-recombination line denoted as $\mathrm{AX}^{+} \mathrm{CR}$ was observed. It was named as cyclotron resonance line being similar by its spectral position to the exciton-cyclotron absorption line detected in Ref. [17] and discussed in Ref. [18]. The emission line $\mathrm{AX}^{+} \mathrm{CR}$ is represented in Fig. 7 reproduced from Ref. [10]. At small values of the magnetic field strength $H$ the line $\mathrm{AX}^{+} \mathrm{CR}$ is similar with the usual SU line, but with the increasing of $H$ its spectral position shifts continuously towards the high energy side crossing consecutively all trion and exciton lines becoming the highest energy line in the PL spectrum [10]. We suppose that this line will change nonmonotonously at very high magnetic fields as it is represented in Fig. 7 and as one can expect taking into account the evolution of some heavy hole energy

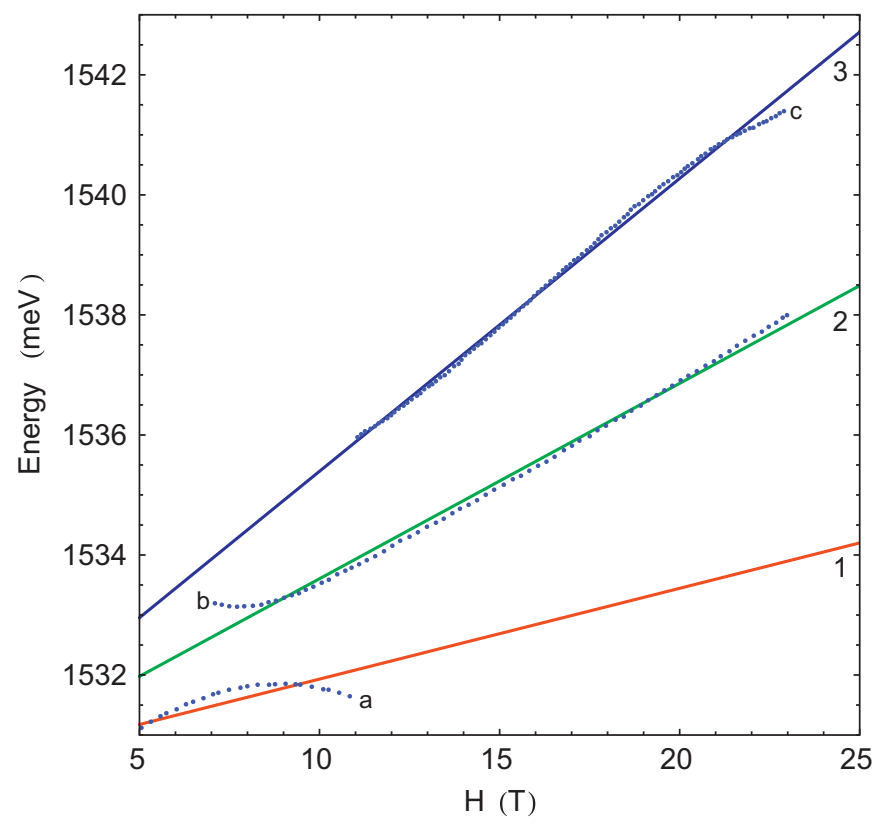

Fig. 6. The dotted lines (a, b, c) represent the experimental data reproduced from Fig. 3 of Ref. [8]. The solid lines 3, 2, 1 represent our approximations of the experimental data, being interpreted as $\mathrm{AX}^{+}, \mathrm{AX}^{+} \mathrm{SU}_{1}$ and $\mathrm{AX}^{+} \mathrm{SU}_{2}$ correspondingly in the frame of the usual Landau quantization without Rashba SOC. 

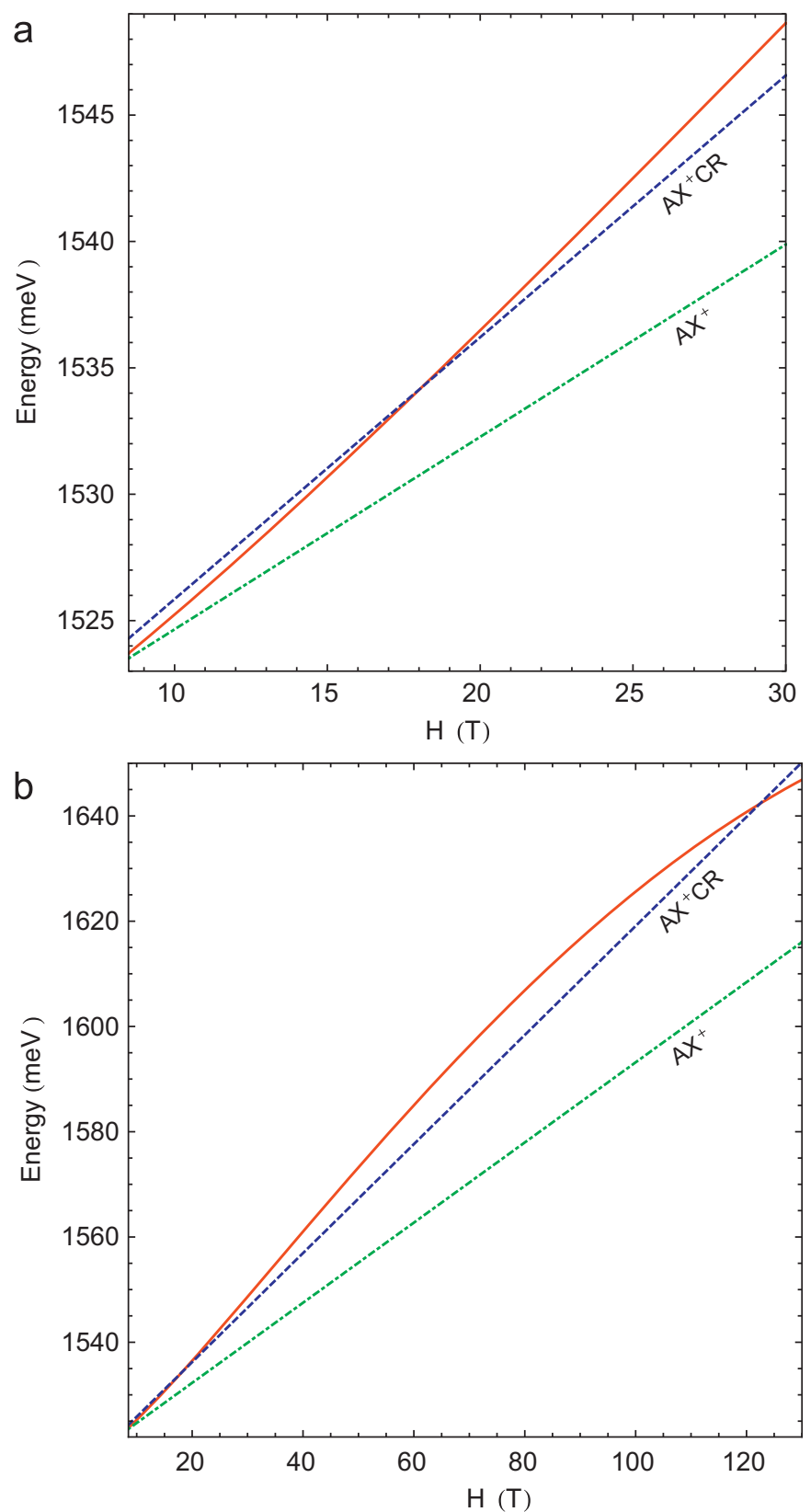

Fig. 7. The emission lines $A X^{+} C R$ and $\mathrm{AX}^{+}$in dependence of the magnetic field strength reproduced from the Fig. 2 of Ref. [10]. First of them is represented by the dashed line and the second one by the dot-dashed line. Our theoretical results are represented by solid line. It represents the SU process at small magnetic fields and $\mathrm{SD}$ process at greater magnetic fields. It reveals the tendency to approach the $\mathrm{AX}^{+}$ line and even to become SU line.

levels represented in Fig. 8. We suppose that such intriguing behavior of the line $\mathrm{AX}^{+} \mathrm{CR}$ is related with the Landau-Rashba quantization of the heavy holes captured inside the $\mathrm{AX}^{+}$complex.

As one can see in Fig. 8 the energy levels have a nonmonotonous dependences on $H$. In the case of the given parameters $C$ and $E_{z}$ in the range of small magnetic fields $H \lesssim 3 \mathrm{~T}$ the lowest energy level has the spinor quantum number $m=3$. The energy levels with spinor quantum numbers $m=4,5,6$ have greater energies. The excitation of the second hole with $m=3$ during the SU recombination process needs energy, which is subtracted from the emitted photon. In the next region of the magnetic fields stretching from $10 \mathrm{~T}$ up till $90 \mathrm{~T}$ the level $m=3$ is higher situated on the energy scale in comparison with the levels $m=4,5,6$. The de-excitations of the holes with $m=3$ and their transitions on the
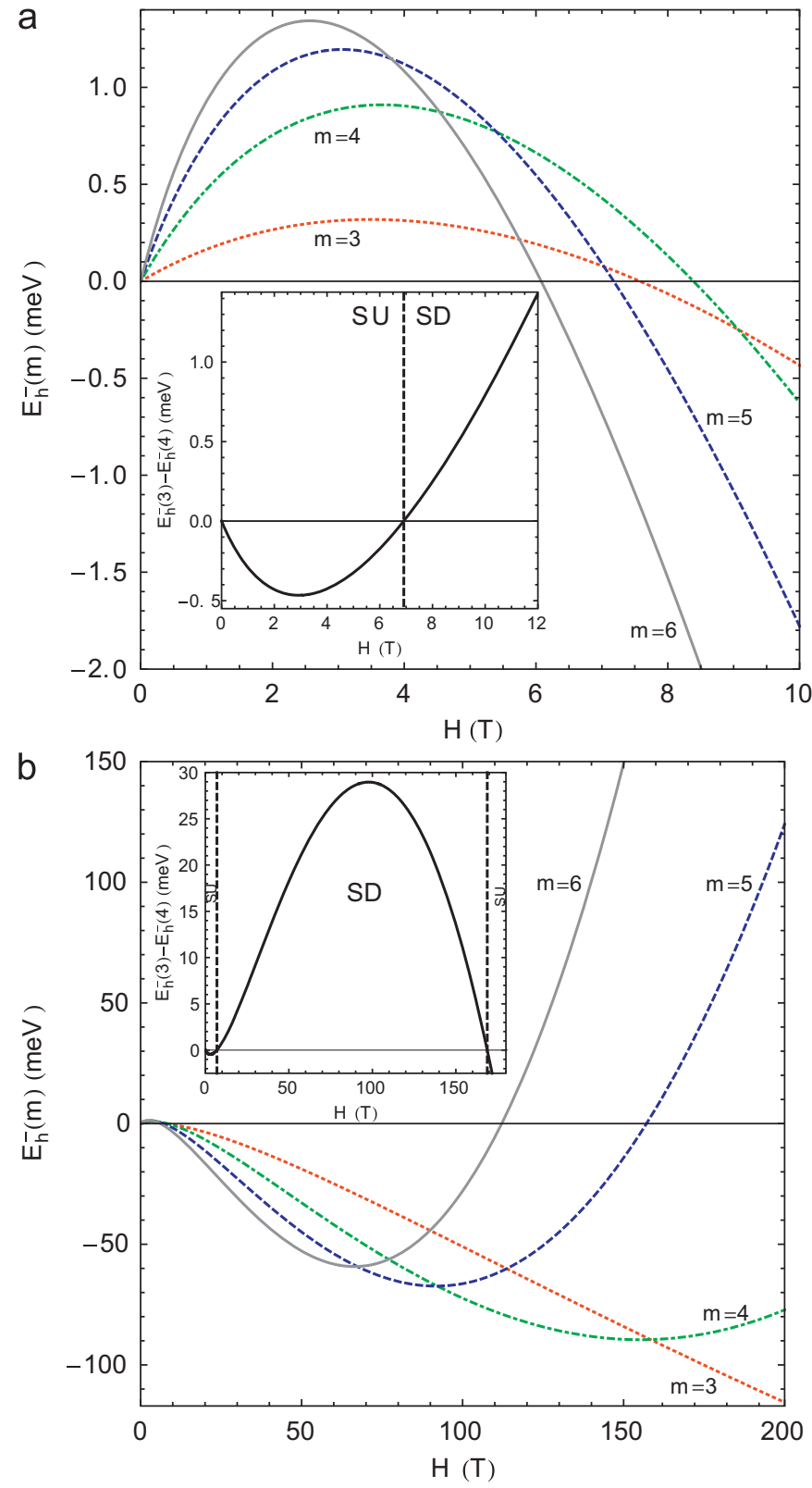

Fig. 8. The heavy hole energy levels $E_{h}^{-}(m)$ with $m=3,4,5,6$ in dependence of the magnetic field strength at another parameters of the theory $C=2.75$ and $E_{z}=7.3 \mathrm{kV} / \mathrm{cm}$. Changing the parameter $E_{z}$ we can influence essentially on the picture of the SU and SD processes. Insets: the difference $E_{h}^{-}(3)-E_{h}^{-}(4)$ at a given parameters $C=2.75$ and $E_{z}=8.1 \mathrm{kV} / \mathrm{cm}$; this difference determines the possibility to obtain the SU and SD processes in the frame of the same Landau levels changing only the magnetic field strength.

levels with $m=4,5,6$ during the Auger-recombination process will be accompanied by the supply of energy. It will increase the energy of the emitted photon and will generate the shake-down process. In the region of the magnetic fields $H \gtrsim 170 \mathrm{~T}$ again the level $m=3$ becomes the lower energy level and the SU process takes place. The continuous evolution of the SU process into SD process and vice versa can be monitored by simple variation of $H$. The concrete realization of such scenario can be achieved if the holes captured inside the $\mathrm{AX}^{+}$complex with the structure $\mathrm{AX}^{+}=\mathrm{A}^{-}+\mathrm{e}+3 \mathrm{~h}$ do exist in the spinor state with $m=3$. One can remember that the spinor wave functions of the LandauRashba states with $m=3$ and 4 have the forms (6), where the state $|n\rangle$ with $n=0,1,3,4$ describe the Landau quantization in Landau gauge description of the spinless particles. The state $n=0$ has the 
smallest radius of the cyclotron orbit. The holes with spinor wave function with $m=3$ have the smallest radii of the Landau quantizations and represent the slimmest spatial blocks in the construction of the complexes $\mathrm{AX}^{+}$. They can be packed in the more compact form being situated as more close possibly to the $\mathrm{A}^{-}$charged acceptor with the more strong Coulomb attraction between the holes and $\mathrm{A}^{-}$center.

The intersections of the Landau levels with the spinor quantum numbers $m=3,4,5,6$ in the range of the magnetic fields (10-20) $\mathrm{T}$ represent for us the most interest. We found out that in that region the level $m=3$ is situated above the Landau levels $m=4,5,6$. As for the other Landau levels with greater numbers, beginning from $m=7$, they are located higher on the energy scale in this region than the level $m=3$. The hole quantum transitions between the Landau levels with very different numbers will have small overlap integrals and vanishing values of transition probability.

The evolution of the $\mathrm{AX}^{+} \mathrm{CR}$ emission line revealed in Ref. [10] is explained as the transformation of the SU process into SD process and vice versa caused by the nonmonotonous dependence on the magnetic field strength $H$ of the hole spinor state with $m=3$.

Side by side with the emission line $\mathrm{AX}^{+} \mathrm{CR}$ discussed above, the same group of investigators in the recent papers $[19,20]$ reported about another emission line CR-AX with the similar intriguing energy-field dependence. The both emission lines $\mathrm{AX}^{+} \mathrm{CR}$ and $\mathrm{CR}-\mathrm{AX}$ do not appear in the symmetric doped samples, but only in the asymmetric doped GaAs QWs. They are present only in the samples subjected to the action of an external electric field applied perpendicularly to the layer surface in addition to the perpendicular magnetic field. It means that the Landau quantization of the charged carriers in the asymmetric samples takes place in the presence of the Rashba spin-orbit coupling, whereas in the case of a symmetric doping it occurs without it. As was argued in Ref. [3] and was remembered above, the third-order chirality terms introduced into the LandauRashba Hamiltonian must be supplemented by the positive quartic term introduced in the heavy hole dispersion law. The both terms are induced by the electric field. The positive term does not permit to the hole Landau levels to penetrate unlimitedly inside the semiconductor energy gap separating the valence and the conduction bands. The penetration is due to the chirality terms and it must be stopped by the positive quartic terms so as to conserve the picture of the semiconductor energy bands. The Landau quantization of the 2D electrons and holes with nonparabolic dispersion laws, with iso-spin components and different chirality terms was proposed in Ref. [4] as a generalization of the Landau [1] and Rashba [2] procedures. Looking at Fig. 1, one may observe that at small magnetic fields the hole Landau level with $m=3$ is situated lower on the energy scale than the Landau levels with $m \geq 4$. In the range of intermediary magnetic fields (8-24 T) their positions are interchanged. It means that the hole transitions from the level $m=3$ to the level $m=4$ in the range of small magnetic field is a shake-up process, whereas in the range of intermediary fields it is a shake-down process. In the first case it is necessary to take energy from the emitted photon due to the electron-hole recombinations inside the acceptor complexes, whereas in the second case the supply of energy is added to the emitted photon.

The existence of the excited holes $\left(h^{*}\right)$ inside the acceptor complexes in the range of intermediary fields is a true conclusion which was suggested in Ref. [19] and can be formulated on the base of Ref. [4]. Its origin is not related with the thermal bath, but with the external electric field.
The emission line $\mathrm{AX}^{+} \mathrm{CR}$ in its moving across the whole energy spectrum from its red side to blue side intersects without mixing all encountered emission lines. In difference on it the emission line CR-AX takes part in the mixing with the emission line $\mathrm{X}_{s}^{+}$giving rise to the cyclotron resonant exciton transfer between the nearly free and strongly localized radiative states following the reaction $\mathrm{h}^{*}+\mathrm{AX}=\mathrm{A}+\mathrm{X}^{+}$.

The energy needed to unbind the exciton from the acceptor was supplied by the excited hole $\left(\mathrm{h}^{*}\right)$ due its shake-down process.

\section{Conclusions}

The intersections, overlappings and degeneracies of the lowest lying Landau levels of the 2D heavy holes in some regions of the magnetic field are possible taking into account their nonparabolic dispersion laws, their spin-orbit coupling and chirality terms. The band-to-band quantum transitions and the exciton energy levels in dependence on the magnetic field strength were determined. The shake-up and shake-down recombination lines with the participation of the acceptor-bound trions $\mathrm{AX}^{+}$were studied.

\section{Acknowledgments}

I.V.P. gratefully acknowledges the Foundation for Young Scientists of the Academy of Sciences of Moldova for the financial support (11.819.05.13F)

\section{References}

[1] L.D. Landau, Zeitschrift für Physik 64 (1930) 629; L.D. Landau, Collection of Papers (in Russian), vol. 1, Nauka, Moscow, 1969, p. 47.

[2] E.I. Rashba, Soviet Physics Fizika Tverdogo Tela (Leningrad) 2 (1960) 1224

[3] T. Hakioğlu, M.A. Liberman, S.A. Moskalenko, I.V. Podlesny, Journal of Physics: Condensed Matter 23 (2011) 345405.

[4] S.A. Moskalenko, I.V. Podlesny, P.I. Khadzhi, B.V. Novikov, A.A. Kiselyov, Solid State Communications 151 (2011) 1690.

[5] K.S. Novoselov, E. McCann, S.V. Morozov, V.I. Fal'ko, M.I. Katsnelson, U. Zeitler, D. Jiang, F. Schedin, A.K. Geim, Nature Physics 2 (2006) 177.

[6] A.H. Castro Neto, F. Guinea, N.M.R. Peres, K.S. Novoselov, A.K. Geim, Reviews of Modern Physics 81 (2009) 109.

[7] S.A. Moskalenko, M.A. Liberman, P.I. Khadzhi, E.V. Dumanov, Ig.V. Podlesny, V.V. Boțan, Physica E 39 (2007) 137.

[8] L. Bryja, A. Wojs, J. Misiewicz, M. Potemski, D. Reuter, A. Wieck, Physical Review B 75 (2007) 035308.

[9] A. Wojs, L. Bryja, J. Misiewicz, M. Potemski, D. Reuter, A. Wieck, Acta Physica Polonica 110 (2006) 429.

[10] J. Jadczak, L. Bryja, P. Plochocka, A. Wojs, J. Misiewicz, D. Maude, M. Potemski, D. Reuter, A. Wieck, Journal of Physics: Conference Series 210 (2010) 012043.

[11] G. Finkelstein, H. Shtrikman, I. Bar-Joseph, Physical Review B 53 (1996) 12593.

[12] S. Glasberg, H. Shtrikman, I. Bar-Joseph, Physical Review B 63 (2001) 201308. (R).

[13] G. Finkelstein, H. Shtrikman, I. Bar-Joseph, Uspekhi Fizicheskikh Nauk 168 (1998) 191.

[14] A.B. Dzyubenko, Physical Review B 69 (2004) 115332.

[15] A.B. Dzyubenko, Physica E 20 (2004) 424.

[16] U. Hergenhahn, A. De Fanis, G. Prümper, A. Kazansky, N.M. Kabachnik, U. Ueda, Physical Review A 73 (2006) 022709.

[17] D.R. Yakovlev, V.P. Kochereshko, R.A. Suris, H. Schenk, W. Ossau, A. Waag, G. Landwehr, P.C.M. Christianen, J.C. Maan, Physical Review Letters 79 (1997) 3974.

[18] S.A. Moskalenko, M.A. Liberman, I.V. Podlesny, Physical Review B 79 (2009) 125425.

[19] L. Bryja, J. Jadczak, A. Wojs, G. Bartsch, D.R. Yakovlev, M. Bayer, P. Plochocka, M. Potemski, D. Reuter, A.D. Wieck, Physical Review B 85 (2012) 165308

[20] J. Jadczak, L. Bryja, A. Wojs, M. Potemski, Physical Review B 85 (2012) 195108. 